\title{
Factorization and soft-gluon divergences in isolated-photon cross sections
}

\author{
S. Catani* \\ Theory Division, CERN, CH 1211 Geneva 23, Switzerland \\ M. Fontannaz \\ LPTHE, Université Paris-Sud, Bâtiment 211, F-91405 Orsay Cedex, France
}

E. Pilon ${ }^{\dagger}$

LAPTH, B.P. 110, F-74941 Annecy-le-Vieux Cedex, France

(Received 23 April 1998; published 2 October 1998)

\begin{abstract}
We study the production of isolated photons in $e^{+} e^{-}$annihilation and give the proof of the all-order factorization of the collinear singularities. These singularities are absorbed in the standard fragmentation functions of partons into a photon, while the effects of the isolation are consistently included in the shortdistance cross section. We compute this cross section at order $\alpha_{S}$ and show that it contains large double logarithms of the isolation parameters. We explain the physical origin of these logarithms and discuss the possibility to resum them to all orders in $\alpha_{S}$.
\end{abstract}

[S0556-2821(98)09119-X]

PACS number(s): $12.38 . \mathrm{Bx}$

\section{INTRODUCTION}

At the CERN $e^{+} e^{-}$collider LEP, the Fermilab Tevatron, and the future CERN Large Hadron Collider (LHC), the detection of high-energy photons produced by short-distance interactions goes through the definition of an isolation criterion that aims at decreasing fake photon signals coming from $\pi^{0}$ decays. The high-energy $\pi^{0}$, which is seen as a single cluster in a calorimeter, belongs to a jet and is accompanied by hadronic energy, whereas a bremsstrahlung photon emitted by a quark is unaccompanied as soon as the photon-quark angle is large enough. Therefore any criterion to select "unaccompanied photons" enhances the signal-to-background ratio.

The criterion that is mostly used in current practice is the following. In $e^{+} e^{-}$collisions at LEP, a photon of energy $E_{\gamma}$ is said to be isolated if it is accompanied by less than a specific amount $\varepsilon_{h} E_{\gamma}$ of hadronic energy $E_{h}^{\text {cone }}$ in a cone of half-angle $\delta$ around the photon momentum. This isolation criterion can be written as follows:

$$
E_{h}^{\text {cone }}=\sum_{i \neq \gamma} E_{i} \Theta\left(\delta-\theta_{i \gamma}\right) \leqslant \varepsilon_{h} E_{\gamma}, \quad \varepsilon_{h}>0,
$$

where the energies and the relative angles $\theta_{i \gamma}$ are defined in the center-of-mass frame. In hadron collisions at the Tevatron, the isolation criterion is similar, but the energies are replaced by transverse energies while the angles and the cone are defined in the azimuth-pseudorapidity plane, so that $\theta_{i \gamma}$ is replaced by $R_{i \gamma}=\sqrt{\eta_{i \gamma}^{2}+\phi_{i \gamma}^{2}}$. Typical values of the isolation parameters $\varepsilon_{h}$ and $\delta$ are $\varepsilon_{h}=\mathcal{O}\left(10^{-1}\right)$ and $\delta \lesssim 0.7$.

\footnotetext{
* On leave of absence from INFN; Sezione di Firenze, Florence, Italy.

†On leave of absence from LPTHE, Université Paris-Sud, Orsay Cedex, France.
}

The criterion in Eq. (1.1) can be stated in an equivalent way by saying that the fraction of electromagnetic energy inside the isolation cone has to be larger than a fixed value $x_{c}:$

$$
\frac{E_{\gamma}}{\sum_{i \neq \gamma} E_{i} \Theta\left(\delta-\theta_{i \gamma}\right)+E_{\gamma}} \geqslant x_{c},
$$

where

$$
x_{c} \equiv \frac{1}{1+\varepsilon_{h}}<1 \text {. }
$$

In the case when no isolation criterion is applied, the inclusive photon cross section is computable by using the QCD factorization formula:

$$
\sigma_{\gamma}=\sum_{A=q, \bar{q}, g, \gamma} C_{A}\left(\mu^{2}\right) \otimes D_{\gamma / A}\left(\mu^{2}\right),
$$

where $\otimes$ denotes the convolution over the energy fraction $z$. The hard-subprocess cross section $C_{A}\left(\mu^{2} ; z\right)$, which describes the production of a high-energy parton $A$, can be calculated order by order in QCD perturbation theory. The nonperturbative phenomena are included in $D_{\gamma / a}\left(z, \mu^{2}\right)$, the inclusive fragmentation function of the QCD parton $a$ ( $a$ $=q, \bar{q}, g)$ into a photon $[1,2]$, and the direct term $(A=\gamma)$ not proportional to the fragmentation function is taken into account through the convention $D_{\gamma / \gamma}\left(z, \mu^{2}\right)=\delta(1-z)$.

In the case of an isolated photon, the isolation criterion (1.1) enforces additional phase-space restrictions. This implies that the cross section is no longer fully inclusive and, hence, that the factorized expression (1.4) is not necessarily valid.

The applicability of the factorization theorem to isolated photons is thus a basic issue that has to be dealt with before the corresponding cross sections can be studied within the 
conventional QCD framework. The perturbative calculations that have been performed so far do not help to this purpose. In the case of hadron collisions, all the available calculations [3] beyond the leading order (LO) in the strong coupling $\alpha_{S}$ are based on approximate methods that, in particular, lead to an incomplete treatment of the fragmentation contributions. The next-to-leading order (NLO) calculation of Ref. [4] for $e^{+} e^{-}$collisions introduces by definition a fragmentation component that explicitly depends on the isolation parameters and thus differs from the process-independent fragmentation function in Eq. (1.4). Actually, in the case of $e^{+} e^{-}$ annihilation, factorization has recently been questioned by Berger, Guo, and Qiu [5]. The results of Ref. [5] have been criticized by some of us in Ref. [6].

In the present paper we confirm that factorization is fulfilled at NLO [6] and extend this argument by proving the validity of factorization to all orders in perturbation theory.

Although we show that isolation does not spoil factorization, yet the phase-space restrictions due to the isolation criterion (1.1) are not harmless, as discussed in detail in the second part of the paper. The factorization theorem deals with collinear singularities that occur in the calculation at the parton level. Once these singularities, whose origin is nonperturbative, have been absorbed in the fragmentation functions, the short-distance cross section can still have a divergent behavior at some points of the phase space when computed order by order in perturbation theory [7]. These divergences are due to certain kinematical constraints that, limiting the fully inclusive character of the cross section, produce an imperfect compensation between real and virtual emission of soft (and collinear) partons. In isolated-photon cross sections, the soft-gluon divergences are double logarithmic and appear at a specific point inside the phase space $[5,6]$. Owing to its perturbative origin, this problem can be solved by summing the logarithmic divergences to all orders in perturbation theory.

The outline of the paper is as follows. In Sec. II we give our proof of the validity of the factorization theorem for isolated-photon cross sections defined by the criterion ${ }^{1}$ in Eq. (1.1). We show how the effects of the isolation are consistently included in the short-distance subprocess and, in particular, we discuss the functional dependence of the shortdistance cross section on the isolation parameters. In Sec. III we consider isolated photons produced in $e^{+} e^{-}$annihilation and we compute the NLO contribution to the fragmentation component of the short-distance cross section. We present results in analytic form for any value of the isolation parameters $\varepsilon_{h}, \delta$. Using these explicit expressions, in Sec. IV we discuss in detail the physical origin of the soft-gluon divergent behavior of the NLO cross section in the vicinity of point $x_{\gamma}=x_{c}$. Finally, in Sec. $\mathrm{V}$ we summarize our results and outline how an all-order resummation can eventually lead to well-behaved theoretical predictions for the cross section.

\footnotetext{
${ }^{1}$ The case of jets containing isolated photons has been considered in Refs. $[4,8,9]$. An alternative definition of the isolated photon has recently been suggested by Frixione [10].
}

\section{FACTORIZATION}

The factorization issue regards both hadron and photon distributions. Thus we make no distinction between these two cases, although isolated-hadron cross sections are of less experimental interest.

\section{A. $e^{+} e^{-}$annihilation}

The customary factorization formula for the inclusive distribution of a single particle $H$ with four-momentum $p_{\gamma}$ produced in $e^{+} e^{-}$annihilation is

$$
\begin{aligned}
\frac{1}{\sigma_{0}} \frac{d \sigma\left(Q^{2}, x_{\gamma}\right)}{d x_{\gamma}}= & \sum_{A} \int_{x_{\gamma}}^{1} \frac{d x}{x} D_{H / A}\left(x_{\gamma} / x, \mu^{2}\right) \\
& \times C_{A}^{\text {fulll) }}\left(\alpha_{s}\left(\mu^{2}\right), Q^{2} / \mu^{2} ; x\right)+\mathcal{O}\left((1 / Q)^{p}\right),
\end{aligned}
$$

where the sum extends over $A=q_{f}, \bar{q}_{f}, g$ when the observed particle $H$ is a hadron and over $A=q_{f}, \bar{q}_{f}, g, \gamma$ when $H=\gamma$ is a photon [in this case $D_{\gamma / \gamma}\left(z, \mu^{2}\right)=\delta(1-z)$, by definition]. The term $\mathcal{O}\left((1 / Q)^{p}\right)$ on the right-hand side denotes corrections that are suppressed by some power $p \geqslant 1$ of the centerof-mass energy $Q$ when $Q \gg \Lambda_{\mathrm{QCD}}$.

Formula (2.1) states that at large values of $Q^{2}$ and for any fixed value of the energy fraction $x_{\gamma}=2 p_{\gamma} \cdot Q / Q^{2}$, all longdistance physics phenomena can be absorbed in the nonperturbative fragmentation function $D_{H / A}\left(z, \mu^{2}\right)$ of the parton $A$ into the particle $H$. The remaining coefficient function $C_{A}^{\text {(full) }}$ $\left(\alpha_{S}\left(\mu^{2}\right), Q^{2} / \mu^{2} ; x\right)$ is short-distance dominated and depends only on the partonic subprocess.

The predictivity of Eq. (2.1) within perturbation theory follows from the fact that not only $C_{A}^{(\text {full) }}$ but also the $Q^{2}$ evolution of the fragmentation functions is perturbatively computable as power-series expansions in $\alpha_{S}$. After having extracted $D_{H / A}\left(z, Q_{0}^{2}\right)$ from experimental data at a certain value $Q^{2}=Q_{0}^{2}$, perturbative QCD predicts the cross section in Eq. (2.1) for any other value of $Q^{2}$.

The content of the factorization theorem in perturbation theory is nonetheless wider. It states that similar factorization formulas are valid for other less inclusive observables and that, in these formulas, the dominant nonperturbative contribution is universal and accounted for by the same fragmentation functions $D_{H / A}\left(z, \mu^{2}\right)$ as in Eq. (2.1). Thus the factorization formulas are obtained from Eq. (2.1) by the replacement

$$
C_{A}^{(\text {full })}\left(\alpha_{s}\left(\mu^{2}\right), Q^{2} / \mu^{2} ; x\right) \rightarrow C_{A}\left(\alpha_{S}\left(\mu^{2}\right), Q^{2} / \mu^{2} ; x,\{J\}\right),
$$

where $\{J\}$ denotes the dependence on the particular observable. The differences among the various observables only regard the perturbatively computable coefficient functions $C_{A}$ and the size and the power $p$ of the power-suppressed corrections.

A particular class of observables to which the factorization theorem applies is the class formed by what we call jet-type observables. These observables can be easily identified by examining how they are defined (and measured) in 
terms of the momenta of the final-state particles in the process. The definition has to fulfill the requirements of (i) infrared safety, (ii) collinear safety, and (iii) collinear factorizability.

The first two requirements regard the dependence on all particle momenta but the triggered momentum $p_{\gamma}$ : infrared safety means that the value of the observable is independent of the momenta of arbitrarily soft particles, and collinear safety implies that, when some final-state particles are produced collinearly, the value of the observable depends on their total momentum rather than on the momentum of each of them. Collinear factorizability means that from the measurement of the observable one cannot distinguish whether the triggered momentum $p_{\gamma}$ is carried by the particle $H$ or by that particle accompanied by a bunch of particles parallel to it.

These properties can be stated in a formal way as follows $[11,12,13]$. Let us first introduce the exclusive cross section $d \sigma_{n}^{(\mathrm{excl})}\left(p_{\gamma}, p_{1}, \ldots, p_{n}\right)$ to produce $n+1$ particles with momenta $p_{\gamma}, p_{1}, \ldots, p_{n}$, so that the single-particle inclusive distribution in Eq. (2.1) can be written in the following form:

$$
\begin{aligned}
\frac{d \sigma\left(Q^{2}, x_{\gamma}\right)}{d x_{\gamma}}= & \sum_{n} \int_{\Omega\left(p_{\gamma}, p_{1}, \ldots, p_{n}\right)} d \sigma_{n}^{(\mathrm{excl})}\left(p_{\gamma}, p_{1}, \ldots, p_{n}\right) \\
& \times \delta\left(x_{\gamma}-2 p_{\gamma} \cdot Q / Q^{2}\right)
\end{aligned}
$$

where the integration extends over the full $(n+1)$-particle phase space $\Omega\left(p_{\gamma}, p_{1}, \ldots, p_{n}\right)$. According to this notation, any less inclusive cross section $d \sigma_{J}$ is given by

$$
\begin{aligned}
\frac{d \sigma_{J}\left(Q^{2}, x_{\gamma}\right)}{d x_{\gamma}}= & \sum_{n} \int_{\Omega\left(p_{\gamma}, p_{1}, \ldots, p_{n}\right)} d \sigma_{n}^{(\mathrm{excl})}\left(p_{\gamma}, p_{1}, \ldots, p_{n}\right) \\
& \times \delta\left(x_{\gamma}-2 p_{\gamma} \cdot Q / Q^{2}\right) F_{J}^{(n)} \\
& \times\left(Q, p_{\gamma},\{J\} ; p_{1}, \ldots, p_{n}\right)
\end{aligned}
$$

where, for any exclusive final state with $n+1$ particles of momenta $p_{\gamma}, p_{1}, \ldots p_{n}$, we have denoted by

$$
F_{J}^{(n)}\left(Q, p_{\gamma},\{J\} ; p_{1}, \ldots, p_{n}\right)
$$

the measurement function that defines the actual observable.

In terms of this function, the properties of a jet-type observable are $^{2}$

(i) infrared safety

$$
\begin{aligned}
F_{J}^{(n+\mathbf{1})}\left(Q, p_{\gamma},\{J\} ; p_{1}, \ldots, \boldsymbol{p}_{i}, \ldots, p_{n+1}\right) \\
\stackrel{p_{i} \rightarrow 0}{\longrightarrow} F_{J}^{(n)}\left(Q, p_{\gamma},\{J\} ; p_{1}, \ldots, p_{n+1}\right),
\end{aligned}
$$

(ii) collinear safety

$$
F_{J}^{(n+\mathbf{1})}\left(Q, p_{\gamma},\{J\} ; p_{1}, \ldots, \boldsymbol{p}_{i}, \boldsymbol{p}_{j}, \ldots, p_{n+1}\right)
$$

${ }^{2}$ In Eqs. (2.6) - (2.8) boldface characters are used just to emphasize the differences between left- and right-hand sides.

$$
\underset{p_{i} \| p_{j}}{\longrightarrow} F_{J}^{(n)}\left(Q, p_{\gamma},\{J\} ; p_{1}, \ldots, \boldsymbol{p}_{i}+\boldsymbol{p}_{j}, \ldots, p_{n+1}\right)
$$

and (iii) collinear factorizability

$$
\begin{aligned}
F_{J}^{(n+1)}\left(Q, \boldsymbol{p}_{\gamma},\{J\} ; p_{1}, \ldots, \boldsymbol{p}_{i}, \ldots, p_{n+1}\right) & \\
& \underset{p_{i} \| p_{\gamma}}{\longrightarrow} F_{J}^{(n)}\left(Q, \boldsymbol{p}_{\gamma}+\boldsymbol{p}_{i},\{J\} ; p_{1}, \ldots, p_{n+1}\right) .
\end{aligned}
$$

In the case of the isolated-particle distribution, the measurement function is specified by the isolation criterion in Eq. (1.1). One sees that the expression (1.1) satisfies the properties (2.6) and (2.7), provided the isolation parameter $\varepsilon_{h}$ is kept finite. ${ }^{3}$

The satisfaction of the collinear factorizability in Eq. (2.8) may appear more problematic by naive inspection of Eq. (1.1). If the isolation parameters $J=\left\{\varepsilon_{h}, \delta\right\}$ were assumed to be the relevant variables for the short-distance subprocess, the measurement function could be defined as

$$
\begin{aligned}
F^{(n+1)}\left(Q, p_{\gamma}, J\right. & \left.=\left\{\varepsilon_{h}, \delta\right\} ; p_{1}, \ldots, p_{n+1}\right) \\
& =\Theta\left(\varepsilon_{h} E_{\gamma}-\sum_{\substack{i=1 \\
i \neq \gamma}}^{n+1} E_{i} \Theta\left(\delta-\theta_{i \gamma}\right)\right) .
\end{aligned}
$$

Then, considering the collinear limit where, for instance, $p_{n+1}$ is parallel to $p_{\gamma}$,

$$
\begin{aligned}
& F^{(n+1)}\left(Q, p_{\gamma}, J\right.\left.=\left\{\varepsilon_{h}, \delta\right\} ; p_{1}, \ldots, p_{n+1}\right) \\
& \underset{p_{n+1} \| p_{\gamma}}{\longrightarrow} \Theta\left(\varepsilon_{h} E_{\gamma}-E_{n+1}-\sum_{\substack{i=1 \\
i \neq \gamma}}^{n} E_{i} \Theta\left(\delta-\theta_{i \gamma}\right)\right) \\
&=F^{(n)}\left(Q, p_{\gamma}-p_{n+1} / \varepsilon_{h}, J=\left\{\varepsilon_{h}, \delta\right\} ; p_{1}, \ldots, p_{n}\right) .
\end{aligned}
$$

Since the function $F^{(n)}$ on the right-hand side of Eq. (2.10) depends on the momentum $p_{\gamma}-p_{n+1} / \varepsilon_{h}$ rather than on $p_{\gamma}$ $+p_{n+1}$, Eq. (2.8) is not satisfied by the definition in Eq. (2.9).

This effect can easily be understood. In its collinearfragmentation process the triggered parton (particle) turns out to be necessarily less isolated (its energy decreases while the amount of accompanying hadronic energy increases) and, eventually, the isolation criterion can be violated. Thus one cannot insist on factorizing a short-distance subprocess that depends on the fixed isolation parameter $\varepsilon_{h}$. Hard partons produced at short distances have to be more isolated than the triggered particle $H$, and their isolation has to be increased as the energy of $H$ decreases.

\footnotetext{
${ }^{3}$ The violation of infrared safety in the case of perfect isolation, i.e., when $E_{h}^{\text {cone }}=0$, was pointed out in Refs. $[4,8,14]$.
} 
This is the key point, to show that the isolated cross section is a jet-type observable. The isolation criterion in Eq. (1.1) can indeed be recast in a form that explicitly satisfies collinear factorizability by considering $J=\left\{r_{\gamma}, \delta\right\}$ as the relevant parameters of the hard-scattering subprocess. Here $r_{\gamma}$ is defined by

$$
r_{\gamma} \equiv \frac{x_{\gamma}}{x_{c}} \geqslant \frac{2\left(\sum_{i \neq \gamma} E_{i} \Theta\left(\delta-\theta_{i \gamma}\right)+E_{\gamma}\right)}{Q}>x_{\gamma},
$$

and represents the upper limit on the total-energy fraction inside the isolation cone. Using these isolation parameters, the measurement function that corresponds to the criterion in Eq. (1.1) can be written as

$$
\begin{aligned}
F^{(n)}\left(Q, p_{\gamma}, J\right. & \left.=\left\{r_{\gamma}, \delta\right\} ; p_{1}, \ldots, p_{n}\right) \\
& =\Theta\left(r_{\gamma} \frac{Q}{2}-\left[\sum_{\substack{i=1 \\
i \neq \gamma}}^{n} E_{i} \Theta\left(\delta-\theta_{i \gamma}\right)+E_{\gamma}\right]\right) .
\end{aligned}
$$

Equation (2.8), as well as Eqs. (2.6) and (2.7), straightforwardly applies to the function in Eq. (2.12) as long as $r_{\gamma}$ $>2 E_{\gamma} / Q=x_{\gamma}$. Note that, performing the limits of Eqs. (2.6)-(2.8), the parameters $J=\left\{r_{\gamma}, \delta\right\}$ in Eq. (2.12) have to be kept fixed and regarded as variables that are independent of the momenta $p_{\gamma}, p_{1}, \ldots, p_{n}$. In particular, this implies that the factorized coefficient functions of Eq. (2.2) explicitly depend on the parameters $J=\left\{r_{\gamma}, \delta\right\}$.

We thus conclude that, for the production cross section of isolated photons, factorization is valid and the all-order factorization formula is

$$
\begin{aligned}
\frac{1}{\sigma_{0}} \frac{d \sigma\left(Q^{2}, x_{\gamma}, x_{c}, \delta\right)}{d x_{\gamma}}= & \sum_{a=g_{f}, \bar{q}_{f}, g} \int_{x_{\gamma}}^{1} \frac{d x}{x} D_{\gamma / a}\left(x_{\gamma} / x, \mu^{2}\right) \\
& \times C_{a}\left(\alpha_{s}\left(\mu^{2}\right), Q^{2} / \mu^{2} ; x, r_{\gamma}, \delta\right) \\
& +C_{\gamma}\left(\alpha_{s}\left(\mu^{2}\right), Q^{2} / \mu^{2} ; x_{\gamma}, r_{\gamma}, \delta\right),
\end{aligned}
$$

where $C_{a}$ and $C_{\gamma}$ are the coefficient functions for the fragmentation and direct components, respectively. Note that the fragmentation function $D_{\gamma / a}$ is independent of the isolation parameters $\delta$ and $\varepsilon_{h}$. The effect of the isolation is entirely included in $C_{a}$ and $C_{\gamma}$ through their dependence on $\delta$ and $r_{\gamma}=x_{\gamma} /\left(1+\varepsilon_{h}\right)$.

A final remark is in order. At present, a field-theory proof of the factorization theorem is known only for fully inclusive deep inelastic scattering (DIS). In the case of less inclusive observables, factorization can be justified order by order in perturbation theory on the basis of general power-counting arguments [15]. The requirements of infrared and collinear safety and collinear factorizability are sufficient to guarantee the validity of factorization through power counting. In this respect, our proof of factorization for isolated-photon cross sections cannot be considered rigorous from a field-theory viewpoint, but it is certainly at the same level of rigor as for any other semi-inclusive cross sections [15].

\section{B. Hadroproduction and photoproduction collisions}

The discussion of the previous subsection can straightforwardly be extended to the production of isolated photons in collisions of hadrons and/or real photons. In these cases, the calculation of the cross section at the parton level contains additional singularities that are produced by initial-state collinear radiation. These singularities have to be absorbed in the nonperturbative parton distributions of the colliding particles. Thus the essential difference with respect to $e^{+} e^{-}$ annihilation is that, in hadronic collisions, the photonisolation criterion must not spoil the factorization of the initial-state collinear singularities.

As mentioned in Sec. I, in current experimental practice [16] the hadronic-collision versions of the isolation criterion (1.1) involve transverse energies and angular distances evaluated in the azimuth-pseudorapidity plane. These variables are invariant under longitudinal boosts along the beam direction and, hence, they are insensitive to initial-state collinear radiation. It follows that these types of isolation criteria satisfy the QCD factorization theorem.

Note, however, that the factorization of initial-state collinear singularities can be easily violated when boostnoninvariant variables are used. For instance, this is the case if one considers the angular distances $R_{i \gamma}=\sqrt{\eta_{i \gamma}^{2}+\phi_{i \gamma}^{2}}$ and insists on using energies rather than transverse energies.

\section{FIXED-ORDER CALCULATION IN $e^{+} e^{-}$ANNIHILATION}

To explicitly check the factorization formula (2.13) for $e^{+} e^{-}$annihilation, one needs a perturbative calculation to (at least) the first nontrivial order in $\alpha_{S}$. We have carried out this calculation in analytic form, and the results are presented in this section. We limit ourselves to the case in which the isolation parameters $\varepsilon_{h}, \delta$ vary in the range of practical interest: namely,

$$
0<\varepsilon_{h} \leqslant 1, \quad 0<\delta \leqslant \pi / 2 .
$$

The coefficient functions $C_{a}$ for the fragmentation component of Eq. (2.13) have the following perturbative expansions:

$$
C_{a}\left(\alpha_{s}\left(\mu^{2}\right), Q^{2} / \mu^{2} ; x, r_{\gamma}, \delta\right)=\lambda_{a}\left[C_{a}^{(\mathrm{LO})}\left(x, r_{\gamma}\right)+\frac{\alpha_{s}\left(\mu^{2}\right)}{2 \pi} C_{a}^{(\mathrm{NLO})}\left(Q^{2} / \mu^{2} ; x, r_{\gamma}, \delta\right)\right]+\mathcal{O}\left(\alpha_{s}^{2}\right),
$$

where $\lambda_{q_{f}}=\lambda_{\bar{q}_{f}}=e_{q_{f}}^{2}, \lambda_{g}=\Sigma_{f} e_{q_{f}}^{2}, C_{q_{f}}=C_{\bar{q}_{f}}=C_{q}$, and $e_{q_{j}}$ is the electric charge of the quark of flavor $f$. 
At LO only the quark coefficient function contributes:

$$
C_{q}^{(\mathrm{LO})}\left(x, r_{\gamma}\right)=\delta(1-x) \Theta\left(r_{\gamma}-1\right), \quad C_{g}^{(\mathrm{LO})}\left(x, r_{\gamma}\right)=0 .
$$

Note also that $C_{q}^{(\mathrm{LO})}\left(x, r_{\gamma}\right)$ is nonvanishing only for $r_{\gamma} \geqslant 1$, i.e., for $x_{\gamma} \geqslant x_{c}$.

The results of our calculation for the NLO expressions can be written as

$$
C_{a}^{(\mathrm{NLO})}\left(Q^{2} / \mu^{2} ; x, r_{\gamma}, \delta\right)=\Theta\left(r_{\gamma}-1\right)\left[C_{a}^{(\mathrm{NLO}, \text { full })}\left(Q^{2} / \mu^{2} ; x\right)+C_{a}^{(\mathrm{NL}, \text { in })}\left(x, r_{\gamma}, \delta\right)\right]+C_{a}^{(\mathrm{NLO}, \text { out })}\left(x, r_{\gamma}, \delta\right),
$$

where $C_{a}^{(\mathrm{NLO}, \text { full) }}\left(Q^{2} / \mu^{2} ; x\right)$ denote the customary NLO coefficient functions $[17,18]$ for the single-particle distribution, i.e., for the case in which no isolation is applied. Their explicit expressions in the modified minimal subtraction $(\overline{\mathrm{MS}})$ factorization scheme are

$$
\begin{gathered}
C_{q}^{(\mathrm{NLO}, \text { full })}\left(Q^{2} / \mu^{2} ; x\right)=C_{F}\left\{\left[\frac{1+x^{2}}{1-x} \ln \frac{(1-x) Q^{2}}{\mu^{2}}-\frac{3}{2} \frac{1}{1-x}\right]_{+}+\frac{1+x^{2}}{1-x} \ln x^{2}+\left(\frac{2}{3} \pi^{2}-\frac{11}{4}\right) \delta(1-x)+\frac{5}{2}-\frac{3}{2} x\right\}, \\
C_{g}^{(\mathrm{NLO}, \text { full })}\left(Q^{2} / \mu^{2} ; x\right)=C_{F} 2 \frac{1+(1-x)^{2}}{x} \ln \frac{(1-x) x^{2} Q^{2}}{\mu^{2}} .
\end{gathered}
$$

The other two terms on the right-hand side of Eq. (3.4) derive from the isolation criterion and do not depend on the factorization scheme. We find the following.

(i) The contributions $C_{a}^{(\mathrm{NLO} \text {, in) }}$ are nonvanishing only in the region

$$
r_{\gamma} \leqslant 1+\tan ^{2} \frac{\delta}{4}
$$

and are given by

$$
\begin{aligned}
C_{q}^{(\mathrm{NLO}, \mathrm{in})}\left(x, r_{\gamma}, \delta\right)= & -\Theta\left(x_{\gamma}^{+}-x\right) \Theta\left(x-x_{\gamma}^{-}\right) C_{F} \\
& \times\left\{\frac{1+x^{2}}{1-x} \ln \frac{\left(1-r_{\gamma}+x\right)(1-x) \tan ^{2}(\delta / 2)}{r_{\gamma}-1}-(4-x)\left[\frac{x-r_{\gamma}}{1-x}+\frac{1}{1-x \sin ^{2}(\delta / 2)}\right]\right\}, \\
C_{q}^{(\mathrm{NLO}, \text { in })}\left(x, r_{\gamma}, \delta\right)= & -\Theta\left(x_{\gamma}^{+}-x\right) \Theta\left(x-x_{\gamma}^{-}\right) C_{F} \\
& \times\left\{2 \frac{1+(1-x)^{2}}{x} \ln \frac{\left(1-r_{\gamma}+x\right)(1-x) \tan ^{2}(\delta / 2)}{r_{\gamma}-1}-4\left[x-r_{\gamma}+\frac{(1-x)}{1-x \sin ^{2}(\delta / 2)}\right]\right\},
\end{aligned}
$$

where

$$
x_{\gamma}^{ \pm} \equiv \frac{r_{\gamma}}{2}\left(1 \pm \sqrt{1-\frac{4\left(r_{\gamma}-1\right)}{r_{\gamma}^{2} \sin ^{2}(\delta / 2)}}\right)
$$

(ii) The contributions $C_{a}^{(\mathrm{NLO} \text {, out) }}$ are nonvanishing only in the region

$$
r_{\gamma}<1
$$

and have the following explicit expressions:

$$
\begin{gathered}
C_{q}^{(\mathrm{NLO}, \mathrm{out})}\left(x, r_{\gamma}, \delta\right)=\Theta\left(r_{\gamma}-x\right) C_{F}\left\{\frac{1+x^{2}}{1-x} \ln \frac{1}{(1-x) \tan ^{2}(\delta / 2)}-\frac{4-x}{2}\left[\frac{1}{1-x}-\frac{1+x \sin ^{2}(\delta / 2)}{1-x \sin ^{2}(\delta / 2)}\right]\right\}, \\
C_{g}^{(\mathrm{NLO}, \text { out })}\left(x, r_{\gamma}, \delta\right)=\Theta\left(r_{\gamma}-x\right) C_{F}\left\{2 \frac{1+(1-x)^{2}}{x} \ln \frac{1}{(1-x) \tan ^{2}(\delta / 2)}-2\left[1-\frac{\left.(1-x)\left[1+x \sin ^{2}(\delta / 2)\right]\right]}{1-x \sin ^{2}(\delta / 2)}\right]\right\},
\end{gathered}
$$


The origin of the various NLO contributions in Eq. (3.4) is easily understood. We are interested in the process $\gamma^{*}$ $\rightarrow q+\bar{q}+g+\gamma$ when one of the QCD partons is collinear to the photon. Owing to this collinear decay, to compute the coefficient function $C_{a}^{(\mathrm{NLO})}\left(Q^{2} / \mu^{2} ; x, r_{\gamma}, \delta\right)$, we simply have to evaluate the cross section ${ }^{4}$ for the three-parton subprocess $\gamma^{*} \rightarrow q+\bar{q}+g$ when the triggered parton $a(a=q, \bar{q}$, or $g)$ carries momentum $p$, parallel to $p_{\gamma}$, and energy fraction $x$ $=2 E / Q$. We denote by $p_{1}, p_{2}$ the momenta of the other two partons and by $x_{1}, x_{2}$ their energy fractions. According to the isolation criterion specified by Eq. (2.12), the corresponding measurement functions is

$$
F^{(2)}\left(Q, p,\left\{r_{\gamma}, \delta\right\} ; p_{1}, p_{2}\right)=\Theta\left(r_{\gamma}-\sum_{i=1,2} x_{i} \Theta\left(\delta-\theta_{i \gamma}\right)-x\right)
$$

To make explicit the factorization of collinear singularities, we rewrite Eq. (3.14) by adding and subtracting a contribution that is independent of the momenta, as follows:

$$
\Theta\left(r_{\gamma}-1\right)+\left[F^{(2)}\left(Q, p,\left\{r_{\gamma}, \delta\right\} ; p_{1} p_{2}\right)-\Theta\left(r_{\gamma}-1\right)\right] .
$$

When inserted into Eq. (2.4) and combined with the virtual correction, the first term in Eq. (3.15) gives exactly [cf. Eq. (2.3)] the fully inclusive contribution $C_{a}^{(\mathrm{NLO} \text {, full })}\left(Q^{2} / \mu^{2} ; x\right)$ to Eq. (3.4).

Then we have to consider the term in the square brackets in Eq. (3.15), which, on the basis of the general factorization argument of Sec. II, is expected to give a nonsingular contribution. To show that, we decompose this term in two parts that correspond to the cases in which one additional parton, either $p_{1}$ or $p_{2}$, is inside the isolation cone $\left(F^{(2, \text { in })}\right)$ and both partons are outside it $\left(F^{(2, \text { out })}\right)$. Using Eq. (3.14), we obtain

$$
\begin{aligned}
{\left[F^{(2)}\left(Q, p,\left\{r_{\gamma}, \delta\right\} ; p_{1}, p_{2}\right)-\Theta\left(r_{\gamma}-1\right)\right] } \\
=F^{(2, \text { in })}\left(Q, p,\left\{r_{\gamma}, \delta\right\} ; p_{1}, p_{2}\right) \\
\quad+F^{(2, \text { out })}\left(Q, p,\left\{r_{\gamma}, \delta\right\} ; p_{1}, p_{2}\right),
\end{aligned}
$$

where

$$
\begin{aligned}
F^{(2, \text { in })}\left(Q, p,\left\{r_{\gamma}, \delta\right\} ; p_{1}, p_{2}\right) & \\
= & -\left\{\Theta\left(\delta-\theta_{1 \gamma}\right)\left[\Theta\left(r_{\gamma}-1\right)-\Theta\left(r_{\gamma}-x_{1}-x\right)\right]\right. \\
& +(1 \leftrightarrow 2)\}, \\
F^{(2, \text { out })}\left(Q, p,\left\{r_{\gamma}, \delta\right\} ; p_{1}, p_{2}\right) & \\
= & \Theta\left(\theta_{1 \gamma}-\delta\right) \Theta\left(\theta_{2 \gamma}-\delta\right)\left[\Theta\left(r_{\gamma}-x\right)-\Theta\left(r_{\gamma}-1\right)\right] .
\end{aligned}
$$

\footnotetext{
${ }^{4}$ More technical details can be found in Ref. [6].
}

Let us first consider the emission inside the cone. Since $x_{1}+x \geqslant 1$ because of kinematics, the term in the square brackets on the right-hand side of Eq. (3.17) corresponds to the phase-space region

$$
x_{1}+x>r_{\gamma} \geqslant 1 \text {. }
$$

This forbids the parton $p_{1}$ from becoming either soft $\left(x_{1}\right.$ $=0)$ or collinear to the photon $\left(\theta_{1 \gamma}=0\right)$ because in both cases the three-parton kinematics implies $x_{1}+x=1$, thus violating the constraint (3.19). Therefore, we can safely perform the integration over $p_{1}$ and obtain the finite contribution $C_{a}^{(\mathrm{NLO}, \text { in) }}\left(x, r_{\gamma}, \delta\right)$ in Eqs. (3.4), (3.8), and (3.9). Note that parton radiation inside the isolation cone is included in both terms $C_{a}^{(\mathrm{NLO} \text {, full) }}$ and $C_{a}^{(\mathrm{NLO} \text {, in) }}$ of Eq. (3.4). The contribution of $C_{a}^{(\mathrm{NLO}, \text { in) }}$ is negative [cf. Eq. (3.17)] and represents the suppression effect of the nonisolated distribution produced by the isolation criterion.

A similar discussion applies to the emission outside the cone. Since $x \leqslant 1$ because of kinematics, the term in the square brackets on the right-hand side of Eq. (3.18) vanishes when $r_{\gamma} \geqslant 1$. Thus the term in the square brackets can be replaced by

$$
1>r_{\gamma} \geqslant x .
$$

In this region, $p_{1}$ and $p_{2}$ cannot become either soft or collinear because in both cases one has $x=1$. Therefore the integration over $p_{1}$ and $p_{2}$ is safe and we obtain the finite contribution $C_{a}^{\text {(NLO, out) }}\left(x, r_{\gamma}, \delta\right)$ in Eqs. (3.4), (3.12), and (3.13). Note that the $C_{a}^{\text {(NLO, out) }}$ is positive, although smaller [cf. the subtraction in Eq. (3.18)] than the full nonisolated contribution $C_{a}^{(\mathrm{NLO} \text {, full) }}$ in the region $r_{\gamma}<1$.

We remind the reader that the coefficient function for the direct component of the factorization formula (2.13) was analytically computed to the lowest order by Kunszt and Trocsanyi [4]. Using our notation, their result can be written as follows:

$$
\begin{aligned}
& C_{\gamma}\left(\alpha_{S}\left(\mu^{2}\right), Q^{2} / \mu^{2} ; x_{\gamma}, r_{\gamma}, \delta\right) \\
& \quad=\frac{\alpha}{2 \pi} \lambda_{g}\left[\frac{1}{C_{F}} C_{g}^{(\mathrm{NLO})}\left(Q^{2} / \mu^{2} ; x_{\gamma}, r_{\gamma}, \delta\right)+\mathcal{O}\left(\alpha_{s}\left(\mu^{2}\right)\right)\right],
\end{aligned}
$$

where $\alpha$ is the fine structure constant and $C_{g}^{(\mathrm{NLO})}\left(Q^{2} / \mu^{2} ; x_{\gamma}, r_{\gamma}, \delta\right)$ is given in Eq. (3.4). The relation (3.21) between $C_{\gamma}$ and our result for the NLO gluon coefficient function $C_{g}^{(\mathrm{NLO})}$ can be regarded as a partial check of the calculation described in this section.

Our NLO results in Eqs. (3.4)-(3.13) do not fully confirm those in Ref. [5]. There, the coefficient functions $C_{a}^{(\mathrm{NLO})}$ were found to be affected by singularities (single and double poles in $1 / \epsilon$, where $\epsilon=4-d$ parametrizes the number $d$ of space-time dimensions in dimensional regularization) that would spoil conventional factorization at the specific point $x_{\gamma}=x_{c}$. The method of calculation used in Ref. [5] and the 
interpretation of these singularities have been criticized in Ref. [6]. The method described in this section clearly exhibits the factorization of the collinear singularities. Using the decomposition in Eq. (3.15), we separate a term (that in the square bracket), which is manifestly free from singularities 5 and computable in four space-time dimensions, from a remaining contribution whose dependence on the isolation parameters is only due to the constraint $r_{\gamma} \geqslant 1$. This is an overall constraint (i.e., it does not act on the partonic variables) and its effect is thus harmless: the ensuing (dimensionally regularized) singularities are the collinear singularities that universally enter the fully inclusive cross section.

\section{DIVERGENT BEHAVIOR FOR $x_{\gamma} \sim x_{c}$}

The NLO coefficient functions $C_{a}^{(\mathrm{NLO})}\left(Q^{2} / \mu^{2} ; x, r_{\gamma}, \delta\right)$ in Eq. (3.4) are well behaved for any value $r_{\gamma} \neq 1$, i.e., both for $x_{\gamma}<x_{c}$ and for $x_{\gamma}>x_{c}$. However, when $r_{\gamma} \rightarrow 1$ they become divergent. The divergent behavior (Fig. 1) at this point $x_{\gamma}$ $=x_{c}$, which we shall call the critical point, can be easily derived from the explicit expressions in Eqs. (3.8), (3.9) and (3.12), (3.13).

(i) When the critical point is approached from below,

$$
x_{\gamma}<x_{c}
$$

we obtain

$$
\begin{aligned}
& C_{q}^{(\mathrm{NLO})}\left(Q^{2} / \mu^{2} ; x, r_{\gamma}, \delta\right) \\
& =\delta(1-x) C_{F}\left\{\ln ^{2}\left[\left(1-r_{\gamma}\right) \tan ^{2}(\delta / 2)\right]\right. \\
& \left.\quad+\frac{3}{2} \ln \left[\left(1-r_{\gamma}\right) \tan ^{2}(\delta / 2)\right]\right\}+\mathcal{O}(1), \\
& C_{g}^{(\mathrm{NLO})}\left(Q^{2} / \mu^{2} ; x, r_{\gamma}, \delta\right)=\mathcal{O}(1) .
\end{aligned}
$$

(ii) When the critical point is approached from above,

$$
x_{c} \leqslant x_{\gamma}
$$

we find

\footnotetext{
${ }^{5}$ By "manifestly free from singularites" we mean the following. If this term is evaluated in $d=4-\epsilon$ space-time dimensions, then the limit $\epsilon \rightarrow 0$ can safely be performed because it leads to the contributions $C_{a}^{(\mathrm{NLO}, \text { in })}\left(x, r_{\gamma}, \delta\right), C_{a}^{(\mathrm{NLO} \text {, out })}\left(x, r_{\gamma}, \delta\right)$ that are integrable in any interval of the kinematical variables $x, r_{\gamma}, \delta$. This integrability guarantees that the perturbative coefficient functions are welldefined distributions. However, it does not imply that they are nonsingular functions (cf. the discussion in Sec. IV).
}

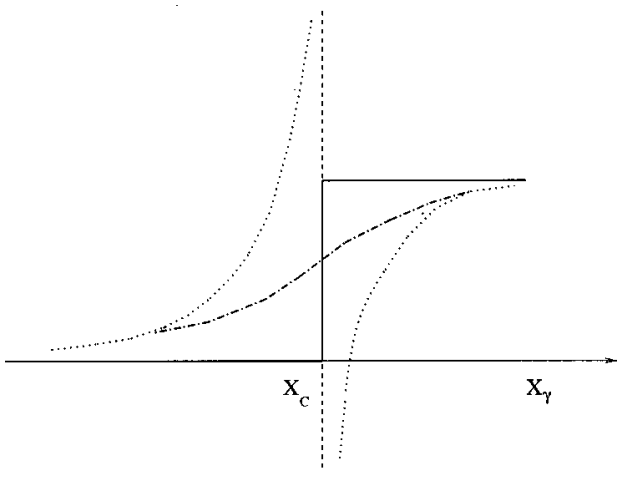

FIG. 1. Divergent behavior of the isolated-photon cross section in the vicinity of the critical point $x_{\gamma}=x_{c}$ at LO (solid line), NLO (dotted line), and as expected after resummation (dot-dashed line).

$$
\begin{aligned}
C_{q}^{(\mathrm{NLO})}\left(Q^{2} / \mu^{2} ; x, r_{\gamma}, \delta\right) \\
=C_{F}\left\{\delta(1-x)\left[-\ln ^{2}\left(\frac{r_{\gamma}-1}{\tan ^{2}(\delta / 2)}\right)-\frac{3}{2} \ln \left(\frac{r_{\gamma}-1}{\tan ^{2}(\delta / 2)}\right)\right]\right. \\
\left.+\left(\frac{1+x^{2}}{1-x}\right)_{+} \ln \left(\frac{r_{\gamma}-1}{\tan ^{2}(\delta / 2)}\right)\right\}+\mathcal{O}(1)
\end{aligned}
$$

$$
\begin{aligned}
& C_{g}^{(\mathrm{NLO})}\left(Q^{2} / \mu^{2} ; x, r_{\gamma}, \delta\right) \\
& \quad=C_{F} 2 \frac{1+(1-x)^{2}}{x} \ln \left(\frac{r_{\gamma}-1}{\tan ^{2}(\delta / 2)}\right)+\mathcal{O}(1),
\end{aligned}
$$

where $\mathcal{O}(1)$ stands for any term that is finite for $r_{\gamma}=1$. Owing to relation (3.21), the coefficient function $C_{\gamma}\left(\alpha_{S}\left(\mu^{2}\right), Q^{2} / \mu^{2} ; x_{\gamma}, r_{\gamma}, \delta\right)$ for the direct component diverges when $x_{c}$ is approached from above.

The presence of these logarithmic divergences was first pointed out in Ref. [5]. It is not in contradiction with the factorization of the collinear singularities [6]. Indeed, these two types of singularities have different physical origins, as discussed in rather general terms in Ref. [7] and recalled below.

The factorization theorem deals with infrared singularities that affect the parton-level calculation for the whole range (or for finite intervals) of the relevant kinematical variables, e.g., $x_{\gamma}, \delta$. These singularities are due to the long-distance component of the scattering process that is intrinsically nonperturbative, in the sense that it is not suppressed by some inverse power of $Q$ as the hard-scattering scale $Q$ increases. The validity of the factorization theorem for the isolatedphoton cross section guarantees that this nonperturbative component can be described by the universal fragmentation function $D_{\gamma / a}\left(x, Q^{2}\right)$. The remaining contributions to the cross section are short-distance dominated. They consist of (i) power-suppressed terms [cf. the term $\mathcal{O}\left((1 / Q)^{p}\right)$ on the right-hand side of Eq. (2.1)] that are controlled by nonperturbative phenomena and of (ii) a short-distance component, 
the coefficient functions $C_{A}$, which depends only logarithmically on $Q$ and is computable as a power series expansion in $\alpha_{S}\left(Q^{2}\right)$.

However, the fact that the coefficient functions are perturbatively computable does not imply that the coefficients of their perturbative expansion are nonsingular functions. In fact, in general these coefficients are singular generalized functions or distributions that lead to finite quantities only when they are integrated with sufficiently smooth test functions. The "plus" distribution

$$
w(x)=2 C_{F}\left[\frac{1}{1-x} \ln \frac{1}{1-x}\right]_{+}
$$

that enters in Eq. (3.5) is a well-known example of this type of singular generalized functions. An analogous divergent behavior, namely,

$$
\begin{aligned}
C_{a}^{(\mathrm{NLO})}( & \left.Q^{2} / \mu^{2} ; x, r_{\gamma}, \delta\right) \\
\simeq & \Theta\left(1-r_{\gamma}\right) \Theta\left(r_{\gamma}-x\right) C_{F} \\
& \times \ln \frac{1}{\tan ^{2}(\delta / 2)} \times\left\{\begin{array}{cc}
\frac{1+x^{2}}{1-x} & (a=q), \\
2 \frac{1+(1-x)^{2}}{x} & (a=g),
\end{array}\right.
\end{aligned}
$$

is observed in the NLO coefficient functions of Eq. (3.4) when the cone size $\delta \rightarrow 0$ at fixed $r_{\gamma}$. The double- and single-logarithmic divergences in Eqs. (4.2), (4.6), and (4.5) are integrable in any neighborhood of the point $x_{\gamma}=x_{c}$ and essentially belong to the same type of singularities [19]. These singularities are known as divergences of the Sudakov type and, in spite of the validity of the factorization theorem, they are still produced by the radiation of soft and/or collinear partons.

The main difference between the divergent behavior in Eqs. (3.5) and (4.8) and that in Eqs. (4.2), (4.5), and (4.6) is that the former appears near the exclusive boundary of the phase space (i.e., $x \rightarrow 1, \delta \rightarrow 0$ ), while the latter occurs at a point $x_{\gamma}=x_{c}$ inside the physical region $0<x_{\gamma}<1$. The Sudakov singularities that arise at an exclusive boundary of the phase space are the most common and extensively studied. ${ }^{6}$ They are due to the loss of balance between the virtual contributions and the radiative tail of the real emission, which is strongly suppressed in this extreme kinematic regime. Sudakov singularities inside the physical region of the phase space have attracted less attention, but are nonetheless quite common in jet physics. Some examples are the $C$-parameter distribution [21] in $e^{+} e$ annihilation and the jet shape [22] in hadron collisions.

The general origin of Sudakov singularities at a critical point inside the physical region has recently been discussed [7]. They arise whenever the observable in question has a

\footnotetext{
${ }^{6}$ See, for instance, Refs. $[19,20]$ and references therein.
}

nonsmooth behavior in some order of perturbation theory at that point. This can happen if the phase-space boundary for a certain number of partons lies inside that for a larger number or if the observable itself is defined in a nonsmooth way. Both mechanisms are responsible for the singular behavior in the case of the isolated-photon cross section. The doubleand single-logarithmic divergences in the square brackets of Eqs. (4.2) and (4.5) are due to the first mechanism, while the remaining single-logarithmic terms in Eqs. (4.5) and (4.6) are due to the second mechanism. We discuss these points in turn.

At LO the phase-space region $r_{\gamma}<1$ is not accessible to the fragmentation component because there is a single parton, the triggered quark, inside the isolation cone. Therefore the coefficient function $C_{q}^{(\mathrm{LO})}\left(x, r_{\gamma}\right)$ in Eq. (3.3) has a step at the critical point $r_{\gamma}=1$. As proved in Ref. [7], this stepwise behavior necessarily produces double-logarithmic singularities at the next order in perturbation theory. When applied to our case, the general argument of Ref. [7] is as follows. The NLO subprocess contains a term that is obtained by radiating a soft (and collinear) gluon from the LO subprocess. This term gives the following contribution to the NLO coefficient function:

$$
C_{q}^{(\mathrm{NLO})}\left(x, r_{\gamma}, \delta\right)=\int_{0}^{1} d x w(z) C_{q}^{(\mathrm{LO})}\left(x, r_{\gamma}(z)\right)+\cdots
$$

where $1-z$ is the energy fraction of the soft gluon $(1-z$ $\ll 1)$ and the ellipsis stands for less singular terms when $r_{\gamma}$ $\rightarrow 1$. In Eq. (4.9), $w(z)$ denotes the probability (in units of $\left.\alpha_{S} / 2 \pi\right)$ of emission of the soft gluon from the triggered quark; it is given by the usual "plus" distribution in Eq. (4.7). The integration over $z$ follows from energy conservation and relates the value of the parameter $r_{\gamma}$ after the emission (i.e., on the left-hand side) to the corresponding value $r_{\gamma}(z)$ before the emission. To explicitly evaluate Eq. (4.9), we have to specify how $r_{\gamma}(z)$ depends on $r_{\gamma}$ and $z$. Since $r_{\gamma}$ is constrained by the fraction of the total energy inside the isolation cone [see Eq. (2.11)], its value decreases $\left[r_{\gamma}\right.$ $\left.<r_{\gamma}^{\text {(out) }}(z)\right]$ or increases $\left[r_{\gamma}>r_{\gamma}^{\text {(in) }}(z)\right]$ according to whether the soft gluon is radiated outside or inside the cone. Neglecting less singular terms ${ }^{7}$ in the soft limit $1-z \ll 1$, we can thus write

$$
\begin{aligned}
& r_{\gamma}^{(\text {out })}(z) \simeq r_{\gamma}+(1-z), \\
& r_{\gamma}^{(\text {in })}(z) \simeq r_{\gamma}-(1-z)
\end{aligned}
$$

Using the explicit expressions (3.3) and (4.7) and inserting Eqs. (4.10) and (4.11) in the integral (4.9), we respectively obtain

\footnotetext{
${ }^{7}$ The actual size of the coefficient in front of the shift $(1-z)$ on the right-hand side of Eqs. (4.10) and (4.11) would affect only the single-logarithmic contributions.
} 


$$
\begin{aligned}
& C_{q}^{(\mathrm{NLO}, \text { out })}\left(x, r_{\gamma}, \delta\right)=2 \delta(1-x) C_{F} \\
& \times \int_{0}^{1} d z\left(\frac{1}{1-z} \ln \frac{1}{1-z}\right)_{+} \\
& \times \Theta\left(r_{\gamma}-z\right)+\cdots \\
& =+\Theta\left(1-r_{\gamma}\right) \delta(1-x) C_{F} \ln ^{2}\left|r_{\gamma}-1\right| \\
& +\cdots, \\
& C_{q}^{(\mathrm{NLO}, \mathrm{in})}\left(x, r_{\gamma}, \delta\right)=2 \delta(1-x) C_{F} \\
& \times \int_{0}^{1} d z\left(\frac{1}{1-z} \ln \frac{1}{1-z}\right)_{+} \\
& \times \Theta\left(r_{\gamma}-1-(1-z)\right)+\cdots \\
& =-\Theta\left(r_{\gamma}-1\right) \delta(-x) C_{F} \ln ^{2}\left|r_{\gamma}-1\right|+\cdots \text {, }
\end{aligned}
$$

in agreement with the double-logarithmic terms in Eqs. (4.2) and (4.5).

Equations (4.9), (4.12), and (4.13) explain the mechanism that produces the logarithmic divergences in the square brackets of Eqs. (4.2) and (4.5). The "plus" prescription in Eq. (4.7) arises from adding real and virtual soft-gluon radiation and, as a result of the cancellation of the soft singularities, leads to finite quantities whenever it acts on smooth functions of $z$. However, this is not the case with Eq. (4.9), because $C_{q}^{(\mathrm{LO})}\left(x, r_{\gamma}(z)\right)$ is discontinuous at $r_{\gamma}(z)=1$. The divergent behavior at the critical point is thus due to the imperfect compensation [5] between real and virtual contributions, which occurs in the presence of the LO-step-like discontinuity at $x_{\gamma}=x_{c}$. Because of the different kinematic recoil in Eqs. (4.10) and (4.11), at NLO the cross section has a double-sided singularity (Fig. 1): that is, it diverges to $+\infty$ and $-\infty$ below and above the critical point $x_{\gamma}=x_{c}$, respectively.

The single-logarithmic term ${ }^{8}$ in Eq. (4.5) (outside the square brackets) and that in Eq. (4.6) have a different origin. They arise from the integration of the collinear spectrum of the parton that is radiated by the triggered parton $a$ at an angle $\theta_{1 \gamma}$ inside the isolation cone. They have indeed the form

$$
P_{q a}(x) \int_{\mu^{2} / Q^{2}}^{\theta_{\max }^{2}} \frac{d \theta_{1 \gamma}^{2}}{\theta_{1 \gamma}^{2}}=P_{q a}(x)\left(\ln \theta_{\max }^{2}+\ln Q^{2} / \mu^{2}\right),
$$

where $P_{q a}(x)$ is the relevant Altarelli-Parisi probability:

\footnotetext{
${ }^{8} \mathrm{We}$ recall that these single-logarithmic divergences appear also in the LO coefficient function $C_{\gamma}$ of the direct component of the cross section.
}

$$
P_{q q}(x)=C_{F}\left(\frac{1+x^{2}}{1-x}\right)_{+}, \quad P_{q g}(x)=C_{F} \frac{1+(1-x)^{2}}{x}
$$

The lower limit of integration over $\theta_{1 \gamma}^{2}$ comes from the factorization of the collinear singularity at $\theta_{1 \gamma}=0$ in the nonperturbative fragmentation function. The upper limit $\theta_{\max }^{2}$ comes from the kinematics of the process. Of course, $\theta_{1 \gamma}$ has to be smaller than the cone size $\delta$. However, when $r_{\gamma} \rightarrow 1$ at fixed $x$ and $\delta$, the energy radiated inside the cone by the splitting process $q \rightarrow a$ can violate the isolation constraint before the cone boundary is actually approached by $\theta_{1 \gamma}$. Therefore $\theta_{\max }^{2} \sim r_{\gamma}-1$ and Eq. (4.14) gives the singlelogarithmic terms of Eqs. (4.5) and (4.6). These terms are due to the nonsmooth character of the isolation criterion, which enforces sharp boundaries on the energies and angles of the radiated partons.

\section{OUTLOOK: HIGHER ORDERS, RESUMMATION, AND NONPERTURBATIVE EFFECTS}

In this paper we have shown that the factorization of collinear singularities in cross sections for the production of isolated particles defined by the criterion (1.1) is valid to any order in QCD perturbation theory. The nonperturbative component of the scattering process that is not power suppressed at high transferred momentum $Q$ is thus taken into account by the universal fragmentation functions $D_{H / a}\left(x, Q^{2}\right)$, whereas the isolation condition is consistently included in the short-distance subprocess.

In the case of isolated photons produced in $e^{+} e^{-}$annihilation, we have checked the factorization pattern by performing an explicit calculation at NLO in $\alpha_{S}$. This calculation shows that, although infrared and collinear safe, the shortdistance component of the NLO cross section has still a divergent behavior (Fig. 1) when the photon energy fraction $x_{\gamma}$ approaches a critical value $x_{c}=1 /\left(1+\varepsilon_{h}\right)$ that is located inside the physical region $0<x_{\gamma}<1$. As shown in Eqs. (4.2), (4.3), (4.5), and (4.6), the divergences are double logarithmic. They are due to the loss of balance between real and virtual contributions that is enforced by the nonsmooth character of the energy-isolation criterion. In Sec. IV we have discussed in detail the physical mechanisms that produce these singularities of the Sudakov type.

The same mechanisms leading to Eqs. (4.2), (4.3), (4.5), and (4.6) will enhance the double-logarithmic divergences by further integer powers of $\ln \left|r_{\gamma}-1\right|$ in yet higher orders of perturbation theory. Since these divergences are unphysical [5], QCD calculations at any finite order in perturbation theory cannot give reliable phenomenological predictions for the isolated-photon cross section in the region around $x_{\gamma}$ $=x_{c}$.

This problem may be overcome by trying to avoid the phase-space region near the critical point. However, also in this case, some general theoretical understanding of the phenomenon is necessary to assess the extent of the dangerous region. The identification of the dangerous region in the perturbative calculation is even more difficult in the case of 


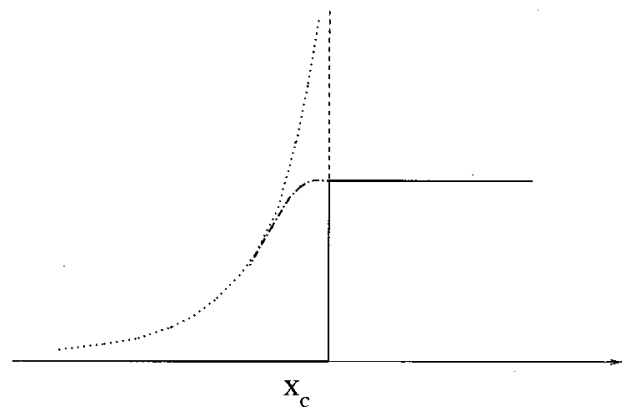

FIG. 2. Divergent behavior at a critical point with single-sided singularities: LO (solid line) and NLO (dotted line). The dotdashed line denotes the Sudakov shoulder obtained after all-order resummation.

hadron collisions [5]. Here the singularities appear at a critical point in the partonic cross section and are smeared by the convolution with the parton distributions of the colliding hadrons.

A better way to deal with the problem is to identify and resum the soft-gluon divergences to all orders in perturbation theory. The resummation approach has been used successfully for the treatment of the Sudakov singularities near the exclusive phase-space boundary for many observables $[19,20]$. The same approach was advocated in Refs. [5, 6] for isolated photons and in Ref. [7] for a general treatment of the Sudakov singularities inside the physical region.

All-order resummation has already been carried out [7] for a particular type of critical point inside the physical region. This type of critical point (Fig. 2) regards cross sections that at $\mathrm{LO}$ have a stepwise behavior at that point and at NLO show a divergence on a single side of the step. After resummation, the cross section is finite, continuous, and differentiable at the critical point. Rather than suppressing the NLO divergence and thus mantaining the steplike behavior, resummation leads to the suppression of the step. The general form of the resummed cross section is a smooth extrapolation from the region where the NLO divergence appears towards the critical point, joining smoothly with the finite value that the cross section has on the other side of the LO step. This characteristic structure was called a Sudakov shoulder [7].

The resummation of the double-sided singularities (Fig. 1) of the isolated-photon cross section is technically more complicated. We expect that it leads to a smooth "jump" structure obtained by smearing (convoluting) two Sudakov shoulders: a shoulder below and an inverted shoulder above the critical point $x_{\gamma}=x_{c}$. As a result, the two sides of the LO step match at some intermediate value at the critical point in the all-order distribution, and the step is smoothed. The actual derivation of the resummed calculation requires more study, since the type of smearing (convolution) to be applied to the two shoulders strongly depends on the detailed isolation kinematics. Work on resummation is in progress, and the results will be reported elsewhere.

Note that the perturbative divergences at $x_{\gamma}=x_{c}$ correspond to integrable singularities and therefore they could in principle be removed by nonperturbative smearing effects as expected, for instance, from hadronization. However, since the hadronization smearing should cancel divergent terms proportional to some power of $\alpha_{S}(Q)$, this would require that the short-distance cross section contain nonperturbative contributions that are not power suppressed at large $Q$. On the basis of our resummation argument, we do not anticipate the presence of these contributions. The resummation of softgluon effects to all orders of perturbation theory should be sufficient to render the isolated-photon cross section finite and smooth throughout the physical phase space. This suggests that the nonperturbative contributions that are not included in the fragmentation function are still powersuppressed.

\section{ACKNOWLEDGMENTS}

We are grateful to Patrick Aurenche and Jean-Philippe Guillet for their collaboration at an early stage of this work. We would like to thank Ed Berger for discussions and comments. This work was supported in part by the EU Fourth Framework Programme "Training and Mobility of Researchers", Network "Quantum Chromodynamics and the Deep Structure of Elementary Particles", Contract No. FMRX-CT98-0194 (DG 12-MIHT).
[1] L. Bourhis, M. Fontannaz, and J. Ph. Guillet, Eur. Phys. J. C2, 529 (1998).

[2] M. Glück, E. Reya, and A. Vogt, Phys. Rev. D 48, 116 (1993); 51, 1427(E) (1995).

[3] P. Aurenche, R. Baier, and M. Fontannaz, Phys. Rev. D 42, 1440 (1990); H. Baer, J. Ohnemus and J. F. Owens, ibid. 42, 61 (1990); E. L. Berger and J. Qiu, ibid. 44, 2002 (1991); B. Bailey, J. Ohnemus, and J. F. Owens, ibid. 46, 2018 (1992); L. E. Gordon and W. Vogelsang, ibid. 50, 1901 (1994).

[4] Z. Kunszt and Z. Trocsanyi, Nucl. Phys. B394, 139 (1993).

[5] E. L. Berger, X. Guo, and J. Qiu, Phys. Rev. Lett. 76, 2234 (1996); Phys. Rev. D 54, 5470 (1996); in QCD and HighEnergy Hadronic Interactions, Proceedings of the 32nd Ren- contres de Moriond, edited by J. Tran Than Van (Editions Frontieres, Paris, 1997), p. 267, hep-ph/9708408.

[6] P. Aurenche, M. Fontannaz, J. Ph. Guillet, A. Kotikov, and E. Pilon, Phys. Rev. D 55, R1124 (1997).

[7] S. Catani and B. R. Webber, JHEP 10, 5 (1997).

[8] G. Kramer and H. Spiesberger, in 'Proceedings of the Workshop on 'Photon radiation from quarks,', Annecy, France, 1991, CERN Report No. 92-04, Geneva, 1992, p. 26; E. W. N. Glover and W. J. Stirling, Phys. Lett. B 295, 128 (1992).

[9] E. W. N. Glover and A. G. Morgan, Z. Phys. C 62, 311 (1994); A. Gehrmann-De Ridder, T. Gehrmann, and E. W. N. Glover, Phys. Lett. B 414, 354 (1997).

[10] S. Frixione, Phys. Lett. B 429, 369 (1998). 
[11] D. E. Soper and Z. Kunszt, Phys. Rev. D 46, 192 (1992); S. Frixione, Z. Kunszt, and A. Signer, Nucl. Phys. B467, 399 (1996).

[12] W. T. Giele and E. W. N. Glover, Phys. Rev. D 46, 1980 (1992); W. T. Giele, E. W. N. Glover, and D. A. Kosower, Nucl. Phys. B403, 633 (1993).

[13] S. Catani and M. H. Seymour, Phys. Lett. B 378, 287 (1996); Nucl. Phys. B485, 291 (1997); B510, 503(E) (1998).

[14] E. L. Berger and J. Qiu, Phys. Lett. B 248, 371 (1990).

[15] J. C. Collins, D. E. Soper, and G. Sterman, in Perturbative Quantum Chromodynamics, edited by A. H. Mueller (World Scientific, Singapore, 1989), p. 1, and references therein.

[16] CDF Collaboration, F. Abe et al., Phys. Rev. Lett. 73, 2662
(1994); D0 Collaboration, S. Abachi et al., ibid. 77, 5011 (1996).

[17] G. Altarelli, R. K. Ellis, G. Martinelli, and S. Y. Pi, Nucl. Phys. B160, 301 (1979).

[18] P. Nason and B. R. Webber, Nucl. Phys. B421, 473 (1994); B480, 755(E) (1996).

[19] S. Catani, in $Q C D$ and High-Energy Hadronic Interactions [5], p. 331, hep-ph/9709503.

[20] G. Sterman, in 10th Topical Workshop on Proton-Antiproton Collider Physics, edited by R. Raja and J. Yoh, AIP Conf. Proc. No. 357 (AIP, New York, 1996), p. 608.

[21] R. K. Ellis, D. A. Ross, and A. E. Terrano, Nucl. Phys. B178, 421 (1981).

[22] M. H. Seymour, Nucl. Phys. B513, 269 (1998). 